\title{
Heat flow reversals without reversing the arrow of time: The role of internal quantum coherences and correlations
}

\author{
C. L. Latune $\odot,{ }^{1,2}$ I. Sinayskiy, ${ }^{1}$ and F. Petruccione $\odot^{1,2,3}$ \\ ${ }^{1}$ Quantum Research Group, School of Chemistry and Physics, University of KwaZulu-Natal, \\ Durban, KwaZulu-Natal 4001, South Africa \\ ${ }^{2}$ National Institute for Theoretical Physics (NITheP), KwaZulu-Natal 4001, South Africa \\ ${ }^{3}$ School of Electrical Engineering, KAIST, Daejeon 34141, Republic of Korea
}

(Received 24 July 2019; published 12 November 2019)

\begin{abstract}
One of the stunning consequences of quantum correlations in thermodynamics is the reversal of the arrow of time, recently shown experimentally in Micadei et al. [Nat. Commun. 10, 2456 (2019)], and manifesting itself by a reversal of the heat flow (from the cold system to the hot one). Here, we show that contrary to what could have been expected, heat flow reversal can happen without reversal of the arrow of time. Moreover, contrasting with previous studies, no initial correlations between system and bath are required. Instead, the heat flow reversal only relies on internal quantum coherences or correlations, which provides practical advantages over previous schemes: One does not need to have access to the bath in order to reverse the heat flow. The underlying mechanism is explained and shown to stem from the collective system-bath coupling and the impact of nonenergetic coherences (coherences between degenerate energy levels) on apparent temperatures. The phenomenon is first uncovered in a broad framework valid for diverse quantum systems containing energy degeneracy. By the end of the paper, aiming at experimental realisations, more quantitative results are provided for a pair of two-level systems. Finally, as a curiosity, we mention that our scheme can be adapted as a correlations-to-energy converter, which have the particularity to be able to operate at constant entropy, similarly to ideal work sources.
\end{abstract}

DOI: 10.1103/PhysRevResearch.1.033097

\section{INTRODUCTION}

Recently, Micadei et al. showed in a two-level atom-based experiment [1] the reversal of the arrow of time thanks to initial correlations between system and reservoir. The macroscopic manifestation of the reversal of the arrow of time is an astonishing heat flow reversal: from the coldest system to the hottest one. Along with the work of Micadei et al., a series of theoretical studies on reversal of the arrow of time [2-7] illustrates one of the most striking consequence of correlations between quantum systems. With a broader perspective, recent years have seen a huge effort toward understanding the role and impact of quantum effects such as correlations and coherences on thermodynamics tasks such as work extraction [8-12], thermal machines [13-20], quantum battery charging [21,22], quantum transport [23-27], and natural or synthetic light-harvesting systems [26,28-34].

Here, we report one more surprising effect of correlations and coherences: heat flow reversal without reversing the arrow of time. One additional major difference with the previous works [1-7] is that in our configuration there is no need for

Published by the American Physical Society under the terms of the Creative Commons Attribution 4.0 International license. Further distribution of this work must maintain attribution to the author $(s)$ and the published article's title, journal citation, and DOI. initial correlations between the system and reservoir, but only coherences (or correlations) within the system (which can be composed of several subsystems). This should simplify its experimental verification and increase its practical interest since it means that the heat flow can be reversed by acting only on the system instead of acting on both the system and reservoir. Moreover, the reported effect is shown to be valid for a large class of systems, the essential ingredients being energy degeneracy and collective coupling. We use the framework introduced in Ref. [35] for the concept of apparent temperature and the role of coherences and correlations in heat flows. The results relevant for this paper are recalled in Sec. II. We also provide an alternative viewpoint using mutual information (Sec. IV), representing an interesting link with previous studies [1,6]. Further in the paper we illustrate the heat flow reversal with a pair of two-level systems (one of the simplest and most experimentally accessible system containing energy degeneracy). While the study in Ref. [36] focused on the energetic and entropic consequences of bath-induced coherences in a pair of two-level systems, the illustration presented in this paper shows how coherences initially present within the pair can lead to curious dynamics such as heat flow reversal. Finally, the scheme can alternatively be used as an intriguing correlations-to-energy converter, which can be tuned to operate at constant entropy, reproducing ideal work source or external (classical) power source [37,38]. As a side note, we mention a study reporting also heat flow reversals but in a different context of topological insulators and topological effects [39]. 


\section{INDISTINGUISHABILITY AND APPARENT TEMPERATURE}

We consider a system $S$ interacting with a thermal bath $B$ at temperature $T_{B}$ (and inverse temperature denoted by $\beta_{B}=$ $\left.1 / T_{B}, k_{B} \equiv 1\right)$. We assume that $S$ can be described by a single energy transition $\omega$ and that $S$ contains energy degeneracy, which includes but is not limited to atoms with degenerate energy levels, such as, for instance, three-level atoms (sometimes referred to as $\Lambda$ and $V$ energy configurations) [40], ensembles of two-level systems, ensembles of spins of arbitrary size, and ensembles of harmonic oscillators. Then, the free Hamiltonian of $S$ has the form $H_{S}=\sum_{n} \sum_{i=1}^{l_{n}} \epsilon_{n}|n, i\rangle\langle n, i|$, where $|n, i\rangle$ is an eigenvector associated to the eigenenergy $\epsilon_{n}$. The index $i$ running from 1 to $l_{n} \geqslant 1$ denotes the degeneracy of the energy level $n$. We consider a coupling between $S$ and the bath of the form

$$
V=g A_{S} B
$$

where $g$ corresponds to the coupling strength, $A_{S}$ is an observable of $S$, and $B$ is an observable of the bath. The energy transitions of $S$ involved in the coupling can be obtained from $A_{S}$ by $\Pi_{n} A_{S} \Pi_{n^{\prime}}$, where $\Pi_{n}$ is the projector onto the eigenspace associated to the eigenenergy $\epsilon_{n}$, which can be expressed in terms of the eigenvectors as

$$
\Pi_{n}=\sum_{i=1}^{l_{n}}|n, i\rangle\langle n, i| .
$$

The form of the coupling (1) implies that all the transitions $\Pi_{n} A_{S} \Pi_{n^{\prime}}$ "see" the same bath. Conversely, the transitions are indistinguishable from the point of view of the bath: An absorption of a bath excitation can activate indiscernibly any resonant transition. We mention this point to emphasize that in general such indistinguishability requires some experimental engineering like parallel transition dipole moments [41] (for atomic systems) or subsystems (if $S$ is an ensemble of subsystems) at spatial locations which are indistinguishable, or indiscernible, from the bath. This last point can usually be obtained by confinement in a volume much smaller than the typical variation length scale of the bath (as, for instance, in superradiance [42-44]) or by adding an ancillary system between the $S$ and the bath to erase part of the information "seen" by the bath as in Refs. [45-48] and experimentally realized, for instance, in Refs. [43,44,49-55].

All transitions $\Pi_{n} A_{S} \Pi_{n^{\prime}}$ of same energy $v=\epsilon_{n^{\prime}}-\epsilon_{n}$ can be put together to form the eigenoperators (or ladder operators) [56] associated to the observable $A_{S}$,

$$
\mathcal{A}(v)=\sum_{\epsilon_{n^{\prime}}-\epsilon_{n}=v} \Pi_{n} A_{S} \Pi_{n^{\prime}}
$$

The observable $A_{S}$ can be rewritten as a sum of its eigenoperators, $A_{S}=\sum_{v} \mathcal{A}(v)$. Since we assumed that $S$ is a single energy transition system (or at least only a single energy transition couples to the bath), the only eigenoperators different from zero are for $v= \pm \omega$. For simplicity, in the remainder of the paper we use the notation, $\mathcal{A}(v=\omega) \equiv$ $\mathcal{A}$ and $\mathcal{A}(v=-\omega) \equiv \mathcal{A}^{\dagger}$. We recall that the eigenoperators satisfy the following commutation relation $\left[H_{S}, \mathcal{A}\right]=-\omega \mathcal{A}$ and $\left[H_{S}, \mathcal{A}^{\dagger}\right]=\omega \mathcal{A}^{\dagger}$. Note that when $S$ is an ensemble of $n$ subsystems $S_{i}$ indistinguishable from the bath, the eigenoperators $\mathcal{A}$ and $\mathcal{A}^{\dagger}$ are the sum of the local eigenoperator $a_{i}$ and $a_{i}^{\dagger}$ of each subsystem $S_{i}, \mathcal{A}=\sum_{i=1}^{n} a_{i}$ and $\mathcal{A}^{\dagger}=\sum_{i=1}^{n} a_{i}^{\dagger}$. In this context, the coupling (1) is a collective coupling between the subsystems $S_{i}$ and the bath. By contrast, an independent coupling of the subsystems with the bath would correspond to a situation where each subsystem $S_{i}$ is coupled to an independent bath operator $B_{i}$ (or even an independent bath).

Under weak coupling between $S$ and the bath, the Markov and Born approximations are valid [56,57] and the reduced dynamics of $S$ is given by a Markovian master equation (see Appendix A). The direction of the heat flow between $S$ and $B$ is given by the sign of energy variation of $S, \dot{E}_{S}:=\operatorname{Tr} \dot{\rho}_{S} H_{S}$. Using the master equation describing the dynamics of $S$, one obtains $\dot{E}_{S} \propto\left(e^{-\omega / T_{B}}-e^{-\omega / \mathcal{T}_{S}}\right)\left(\right.$ see Appendix A), where $\mathcal{T}_{S}$ is defined by [35] $\left(\hbar=1, k_{B}=1\right)$

$$
\mathcal{T}_{S}:=\omega\left(\ln \frac{\left\langle\mathcal{A} \mathcal{A}^{\dagger}\right\rangle}{\left\langle\mathcal{A}^{\dagger} \mathcal{A}\right\rangle}\right)^{-1},
$$

with $\langle\mathcal{O}\rangle:=\operatorname{Tr} \rho_{S} \mathcal{O}$ denoting the expectation value of the operator $\mathcal{O}$ evaluated in the state $\rho_{S}$. The parameter $\mathcal{T}_{S}$ is called the apparent temperature of $S$ since it determines the direction of the heat flow: $\dot{E}_{S}>0\left(\dot{E}_{S}<0\right)$ if and only if $\mathcal{T}_{S}<T_{B}\left(\mathcal{T}_{S}>T_{B}\right)$. Importantly, in the situation where $S$ is an ensemble of subsystems, this result remains valid even if the subsystems are interacting between each other (as long as the interactions conserve the total energy of the ensemble).

Crucially, the apparent temperature $\mathcal{T}_{S}$ takes into account coherences contained in $S$ [35]. This can be seen simply by injecting the expressions of the eigenoperators (3) and of the projectors (2) in the expectation values $\left\langle\mathcal{A} \mathcal{A}^{\dagger}\right\rangle$ and $\left\langle\mathcal{A}^{\dagger} \mathcal{A}\right\rangle$. This yields

$$
\begin{aligned}
\left\langle\mathcal{A} \mathcal{A}^{\dagger}\right\rangle= & \sum_{\epsilon_{n^{\prime}}=\epsilon_{n}=\omega} \sum_{i}^{l_{n}}\left\langle n, i\left|A_{S} \Pi_{n^{\prime}} A_{S}\right| n, i\right\rangle\langle n, i|\rho| n, i\rangle \\
& +\sum_{\epsilon_{n^{\prime}}-\epsilon_{n}=\omega} \sum_{i \neq i^{\prime}}^{l_{n}}\left\langle n, i\left|A_{S} \Pi_{n^{\prime}} A_{S}\right| n, i^{\prime}\right\rangle\left\langle n, i^{\prime}|\rho| n, i\right\rangle,
\end{aligned}
$$

where the first term (5) contains the contribution from the populations $\langle n, i|\rho| n, i\rangle$ of $S$ and the second term (6) contains the contributions from the coherences $\left\langle n, i^{\prime}|\rho| n, i\right\rangle$ between degenerate levels. In the remainder of the paper, such coherences are called nonenergetic coherences as opposed to coherences between nondegenerate levels (levels of different energy) called energetic coherences. Note that the terms in (6) are nonzero if $A_{S}$ contains degenerate transitions (from one level $|n, i\rangle$ to two or more degenerate levels $|m, j\rangle,\left|m, j^{\prime}\right\rangle$ ), which is the essence of the collective or indistinguishable coupling (1) described above. Interestingly, no energetic coherence contributes to the expectation values $\left\langle\mathcal{A} \mathcal{A}^{\dagger}\right\rangle$. The expression of $\left\langle\mathcal{A}^{\dagger} \mathcal{A}\right\rangle$ can be obtained directly from (5) and (6) by replacing $\omega$ with $-\omega$.

It is important to keep in mind that nonenergetic coherences in a many-body system can take the form of correlations between subsystems [35]. For instance, in the simple situation of a pair of two-level systems considered in Sec. V, the term $\chi^{0}=\alpha|0\rangle|1\rangle\left\langle 1\left|\left\langle 0\left|+\alpha^{*}\right| 1\right\rangle\right| 0\right\rangle\langle 0|\langle 1|$ in (16) represents a correlation between the two subsystems (since it implies 
$\rho_{S}^{0} \neq \rho_{S_{1}}^{0} \rho_{S_{2}}^{0}$ ) but it also corresponds to (nonenergetic) coherences between the degenerate states $|01\rangle$ and $|10\rangle$.

Because of the presence of the term (6) in the expectation value of $\mathcal{A} \mathcal{A}^{\dagger}$ and $\mathcal{A}^{\dagger} \mathcal{A}$, it is possible to manipulate the apparent temperature of $S$ only by introducing nonenergetic coherences within $S$. Moreover, when $S$ is a many-body system, correlations between subsystems can correspond to nonenergetic coherences (as just mentioned above) and therefore also affect the apparent temperature $\mathcal{T}_{S}$. In other words, correlated subsystems in an ensemble have an apparent temperature which can largely differ from the apparent temperature of a noncorrelated ensemble but otherwise identical (meaning same local state of each subsystem). In particular, when $S$ is in a thermal state at temperature $T_{S}<T_{B}$ (each subsystem is in a thermal state at temperature $T_{S}$ ), generating correlations between the subsystems can make the resulting apparent temperature $\mathcal{T}_{S}$ larger than $T_{B}$. This suggests that a cold ensemble interacting with a hot bath can appear indeed hotter than the bath and be refrigerated by this hot bath thanks to initial correlations between the subsystems, referred to as internal correlations in the following. Conversely, a hot ensemble can be further heated up by interacting with a cold bath thanks to initial internal correlations. We investigate these curious phenomena in the following.

\section{REVERSING THE HEAT FLOW}

Formalising the above ideas, we consider in this section a many-body system $S$ initially in a state

$$
\rho_{S}^{0}=\rho_{S}^{\text {th }}\left(\beta_{S}\right)+\chi^{0}
$$

composed of a thermal contribution $\rho_{S}^{\text {th }}\left(\beta_{S}\right):=Z^{-1}\left(\beta_{S}\right) e^{-\beta_{S} H_{S}}$ of temperature $T_{S}=1 / \beta_{S}$ and partition function $Z\left(\beta_{S}\right):=$ $\operatorname{Tr} e^{-\beta_{S} H_{S}}$, upgraded by the term $\chi^{0}$ containing arbitrary nonenergetic coherences in form of correlations. Importantly, correlations disappear upon partial trace so that each subsystem of $S$ is locally in a thermal state at temperature $T_{S}$. Therefore, assuming for instance that the bath temperature $T_{B}$ is larger than $T_{S}$, one expects a heat flow from the bath to $S$. This intuitive view omits the role of the correlations between the subsystems. Indeed, the resulting apparent temperature of $S$, given by (4), can be decomposed in the following form:

$$
\frac{\omega}{\mathcal{T}_{S}}=\frac{\omega}{T_{S}}+\log \frac{1+c^{+}}{1+c^{-}},
$$

with $\quad c^{-}:=\left\langle\mathcal{A}^{\dagger} \mathcal{A}\right\rangle_{\mathrm{cor}} /\left\langle\mathcal{A}^{\dagger} \mathcal{A}\right\rangle_{\mathrm{loc}} \quad$ and $\quad c^{+}:=\left\langle\mathcal{A} \mathcal{A}^{\dagger}\right\rangle_{\mathrm{cor}} /$ $\left\langle\mathcal{A} \mathcal{A}^{\dagger}\right\rangle_{\text {loc }}$. The expectation value $\left\langle\mathcal{A} \mathcal{A}^{\dagger}\right\rangle$ has been split into a local contribution $\left\langle\mathcal{A} \mathcal{A}^{\dagger}\right\rangle_{\text {loc }}:=\operatorname{Tr} \rho^{\text {th }}\left(\beta_{S}\right) \mathcal{A} \mathcal{A}^{\dagger}$ corresponding to the term (5) and a contribution from the correlations $\left\langle\mathcal{A} \mathcal{A}^{\dagger}\right\rangle_{\text {cor }}:=\operatorname{Tr} \chi^{0} \mathcal{A} \mathcal{A}^{\dagger}$ corresponding to the term (6). A similar split was made for $\left\langle\mathcal{A}^{\dagger} \mathcal{A}\right\rangle$. Equation (8) shows that the contributions from the correlations add up to the inverse temperature $\beta_{S}$ (corresponding to the local contribution) which can result in an apparent temperature $\mathcal{T}_{S}$ larger than $T_{B}$, implying a heat flow reversal, from $S$ to the bath. The necessary and sufficient conditions for heat flow reversal are

$$
\mathcal{C}>\left\langle\mathcal{A}^{\dagger} \mathcal{A}\right\rangle_{\mathrm{loc}} \frac{e^{\omega \beta_{S}}-e^{\omega \beta_{B}}}{e^{\omega \beta_{B}}-1}>0,
$$

where $\mathcal{C}:=\left\langle\mathcal{A}^{\dagger} \mathcal{A}\right\rangle_{\text {cor }}=\left\langle\mathcal{A} \mathcal{A}^{\dagger}\right\rangle_{\text {cor }}$ (the equality being a direct consequence of $\chi^{0}$ representing correlations between subsystems, see Appendix B) and $\beta_{B}=1 / T_{B}$ is the inverse bath temperature. In other words, the inequality (9) establishes the minimal conditions on the correlations within the ensemble $S$ in order to have a reversal of the heat flow. Since $\mathcal{C}$ is typically limited by a value of the order of $\left\langle\mathcal{A}^{\dagger} \mathcal{A}\right\rangle_{\text {loc }}$ (to ensure the positivity of $\left.\rho^{0}\right)$, one can see from (9) that the heat flow reversal is in general possible only for a limited range of inverse temperatures $\beta_{S}$ around $\beta_{B}$. Moreover, as expected, as $\beta_{S}$ and $\beta_{B}$ become closer, the condition on the correlations weakens.

Conversely, if $T_{S}>T_{B}$, one would expect a heat flow from $S$ to the bath, but again, the contribution from the correlations can reverse the heat flow. This happens if and only if

$$
\mathcal{C}<\left\langle\mathcal{A}^{\dagger} \mathcal{A}\right\rangle_{\mathrm{loc}} \frac{e^{\omega \beta_{S}}-e^{\omega \beta_{B}}}{e^{\omega \beta_{B}}-1}<0
$$

As above, $|\mathcal{C}|$ is limited by a value of the order of $\left\langle\mathcal{A}^{\dagger} \mathcal{A}\right\rangle_{\text {loc }}$ so that the heat flow reversal can happen only for a limited range of inverse temperature $\beta_{S}$ around $\beta_{B}$.

The above considerations can be extended to negative temperatures for $S$ but also for the bath. Indeed, effective baths at negative temperatures (emerging for instance from spin baths [58,59], compositions of thermal baths at different positive temperatures [60], or in a context of thermal machines [61]) are quite common in thermodynamics and play an important role.

Then, when $\beta_{B}$ is negative, heat flow reversals are still possible but the inequality signs in conditions (9) and (10) are inverted.

One other interesting extension of the above picture is considering arbitrary initial state. Then, the thermal state $\rho_{S}^{\text {th }}\left(\beta_{S}\right)$ in the decomposition (7) of the initial state $\rho_{S}^{0}$ is substituted by an arbitrary product of local states of each subsystem of $S$. The above results remain valid by substituting the initial temperature $T_{S}$ by the apparent temperature of the product of the local states.

Additionally, one should keep in mind that one underlying necessary ingredient for the heat flow reversal is the collective coupling (1) introduced in Sec. II. Indeed, when each subsystem interacts with an independent local bath, initial correlations between subsystems cannot be "seen" by local baths so that each subsystem thermalizes to the thermal equilibrium state at the local bath temperature (see also Sec. V A and Fig. 1).

Finally, for the sake of completeness, as suggested in the previous Sec. II, heat flow reversals can be achieved with single system containing nonenergetic coherences. Considering an initial state of the form (7) with $\chi^{0}$ containing nonenergetic coherences and assuming that $T_{B}>T_{S}$ (of arbitrary signs), the conditions for heat flow reversals can be obtained in a similar way as for many-body systems, leading to $\mathcal{C}^{+} e^{\omega \beta_{B}}-$ $\mathcal{C}^{-}>\left\langle\mathcal{A}^{\dagger} \mathcal{A}\right\rangle_{\text {loc }}\left(e^{\omega \beta_{S}}-e^{\omega \beta_{B}}\right)>0$, where $\mathcal{C}^{+}:=\left\langle\mathcal{A} \mathcal{A}^{\dagger}\right\rangle_{\text {coh }} \neq$ $\mathcal{C}^{-}:=\left\langle\mathcal{A}^{\dagger} \mathcal{A}\right\rangle_{\text {coh }}$, with the convention $\langle\mathcal{O}\rangle_{\text {coh }}:=\operatorname{Tr} \chi^{0} \mathcal{O}$, for any operator $\mathcal{O}$. When $T_{B}<T_{S}$, nonenergetic coherences leading to heat flow reversal must satisfy $\mathcal{C}^{+} e^{\omega \beta_{B}}-\mathcal{C}^{-}<$ $\left\langle\mathcal{A}^{\dagger} \mathcal{A}\right\rangle_{\mathrm{loc}}\left(e^{\omega \beta_{S}}-e^{\omega \beta_{B}}\right)<0$. 
In the next section, focusing on many-body systems, we analyze in detail how this heat flow reversal is related to the one reported in Ref. [1]. Interestingly, although both phenomena can be described within the same formalism, they are different in nature. In particular, the present heat flow reversal is not related to reversal of the arrow of time, in contrast to Ref. [1].

\section{DECREASE OF MUTUAL INFORMATION}

The reversal of the arrow of time reported in Ref. [1] relies on the decrease of the mutual information between $S$ and the bath $B$, defined by [62]

$$
I(S: B):=S_{S}+S_{B}-S_{S B},
$$

where $S_{X}:=-\operatorname{Tr}_{X} \rho_{X} \ln \rho_{X}$ denotes the von Neumann entropy and $\rho_{X}$ denotes the density operator of the system $X=S, B, S B$, respectively. Since the mutual information is always positive, being equal to zero only when $S$ and $B$ are uncorrelated [62], a decrease of mutual information $I(S: B)$ is possible if and only if $S$ and $B$ are initially correlated. As a consequence, the entropy production can become negative $[1-3,5,6]$, corresponding to reversing the arrow of time. One of its surprising macroscopic manifestation is a heat flow reversal $[1,5,6]$.

Using the same formalism as in Refs. [1,6], we show that even with $S$ and $B$ initially uncorrelated $[I(S: B)=0]$, one can still have a heat flow reversal. This also provides an alternative point of view on the results of the Sec. III. The key idea is to substitute the mutual bipartite information $I(S: B)$ by the three-partite mutual information $I\left(S_{1}: S_{2}: B\right)$ defined as follows:

$$
I\left(S_{1}: S_{2}: B\right):=S_{S_{1}}+S_{S_{2}}+S_{B}-S_{S B}
$$

Because of the subadditivity of the entropy [62], we have $I\left(S_{1}: S_{2}: B\right) \geqslant I(S: B)$, which guarantees the positivity of $I\left(S_{1}: S_{2}: B\right)$. Then, even if $S$ and $B$ are initially uncorrelated, we can still have $I\left(S_{1}: S_{2}: B\right)>0$ thanks to initial correlations between $S_{1}$ and $S_{2}$. This provides a "fuel" sufficient to reverse the heat flow, as we show in the following.

We derive an expression of $Q$, the heat (exchanged between the initial and final instant of time) from $B$ to $S$, in terms of the variation of the mutual information. We assume that $S$ is initialized in a state of the form (7) that is a product of local thermal states upgraded by some correlations $\chi^{0}$. Since $B$, $S_{1}$, and $S_{2}$ are initially in thermal states, the relative entropy between the initial state $\rho_{X}^{0}$ and the state $\rho_{X}^{t}$ at an arbitrary instant of time $t$ is equal to

$$
\begin{aligned}
S\left(\rho_{X}^{t} \| \rho_{X}^{0}\right) & :=\operatorname{Tr}_{X} \rho_{X}^{t}\left(\log \rho_{X}^{t}-\log \rho_{X}^{0}\right) \\
& =-S_{X}+\beta_{X} E_{X}^{t}+\log Z_{X},
\end{aligned}
$$

where $X$ stands for $S_{1}, S_{2}$, or $B$, and $E_{X}^{t}:=\operatorname{Tr}_{X} \rho_{X}^{t} H_{X}$. From such identity and since $S\left(\rho_{X}^{0} \| \rho_{X}^{0}\right)=0$, one obtains

$$
S\left(\rho_{X}^{t} \| \rho_{X}^{0}\right)=-\Delta S_{X}+\beta_{i} \Delta E_{X},
$$

where $\Delta \mathcal{O}:=\mathcal{O}^{t}-\mathcal{O}^{0}$ is the variation of the quantity $\mathcal{O}=$ $E_{X}, S_{X}$. Finally, assuming that the first law is satisfied (conservation of energy), $\Delta E_{S}=-\Delta E_{B}:=Q$, an expression of the heat exchanged $Q$ can be obtained as follows:

$$
\begin{aligned}
\left(\beta_{S}-\beta_{B}\right) Q= & \beta_{S} \Delta E_{S}+\beta_{B} \Delta E_{B} \\
= & \beta_{S} \Delta E_{S_{1}}+\beta_{S} \Delta E_{S_{2}}+\beta_{B} \Delta E_{B} \\
= & \Delta I\left(S_{1}: S_{2}: B\right)+S\left(\rho_{S_{1}}^{t} \| \rho_{S_{1}}^{0}\right) \\
& +S\left(\rho_{S_{2}}^{t} \| \rho_{S_{2}}^{0}\right)+S\left(\rho_{B}^{t} \| \rho_{B}^{0}\right),
\end{aligned}
$$

where the identity $\Delta E_{S}=\Delta E_{S_{1}}+\Delta E_{S_{2}}$ holds exactly if we assume that $S_{1}$ and $S_{2}$ are not interacting. When $S_{1}, S_{2}$, and $B$ are initially uncorrelated $\left[I\left(S_{1}: S_{2}: B\right)=0\right]$, all the terms in the right-hand side of (15) are positive so that Eq. (15) expresses the "natural" heat flow, from the hottest to the coldest system. However, when $S_{1}$ and $S_{2}$ are initially correlated, the variation of the tripartite mutual information can be rewritten as $\Delta I\left(S_{1}: S_{2}: B\right)=\Delta I\left(S_{1}: S_{2}\right)+\Delta S\left(\rho_{B}\right)$, which can become negative. For $S_{1}$ and $S_{2}$ when highly correlated, the negative contribution from $\Delta I\left(S_{1}: S_{2}\right)$ can even dominate all other positive contributions in the right-hand side of (15), imposing a reversal of the heat flow (when the left-hand side becomes negative). This requires initial correlations between $S_{1}$ and $S_{2}$ to be high enough, which is expressed by Eqs. (9) and (10).

Importantly, the entropy production equal to $[63,64]$ $\Sigma=\Delta I(S: B)+S\left(\rho_{B}^{t} \mid \rho_{B}^{0}\right)$ is always positive, so that the arrow of time is not reversed, in contrast with Ref. [1].

\section{PAIR OF TWO-LEVEL SYSTEMS}

In this section, we focus on the same system considered in Ref. [1], a pair of two-level systems, in order to obtain simple quantitative results which could be verified experimentally. As mentioned in the introduction, the thermodynamic effects of bath-induced coherences in this same system was discussed in Ref. [36]. Here, we focus on a different and somehow opposite aspect: how correlations (or coherences) initially present in the system can dramatically affect both its on going evolution and steady state energy and lead to heat flow reversal. Following the previous sections, we assume that the pair of two-level systems interacts with the bath at inverse temperature $\beta_{B}$ through the collective ladder operators $S^{+}:=\sigma_{1}^{+}+\sigma_{2}^{+}$and $S^{-}:=\sigma_{1}^{-}+\sigma_{2}^{-}$, well known from superradiance [42] and playing the role of $\mathcal{A}^{\dagger}$ and $\mathcal{A}$. The local ladder operators can be expressed in term of the ground and excited states $|0\rangle$ and $|1\rangle$ of each two-level system, namely $\sigma_{i}^{+}:=|1\rangle\langle 0|$ and $\sigma_{i}^{-}=|0\rangle\langle 1|$. We assume that the pair is initially in a state $\rho_{S}^{0}=\rho_{S}^{\text {th }}\left(\beta_{S}\right)+\chi^{0}$ of the form (7), with $H_{S}=\omega \sum_{i=1}^{2} \sigma_{i}^{+} \sigma_{i}^{-}$, $Z\left(\beta_{S}\right)=\left(1+e^{-\omega \beta_{S}}\right)^{2}$, and

$$
\chi^{0}=\alpha|0\rangle|1\rangle\left\langle 1\left|\left\langle 0\left|+\alpha^{*}\right| 1\right\rangle\right| 0\right\rangle\langle 0|\langle 1|
$$

(adopting the convention that the tensor product order is taken to be the same for "bras" and "kets"). Note that we choose $\chi^{0}$ containing only nonenergetic coherences since, as mentioned in Sec. III, energetic coherences do not play any role in the apparent temperature. This choice also corresponds to the initial correlations in Ref. [1].

Applying the results of Sec. III, we have $\mathcal{C}=\operatorname{Tr} \chi{ }^{0} S^{+} S^{-}=$ $2 \operatorname{Re} \alpha \quad\left(\operatorname{Re}\right.$ denotes the real part) and $\left\langle S^{+} S^{-}\right\rangle_{\text {loc }}=$ $\operatorname{Tr} \rho_{S}^{\text {th }}\left(\beta_{S}\right) S^{+} S^{-}=2\left(1+e^{\omega \beta_{S}}\right)^{-1}$, so that a reversal of the 
heat flow happens if and only if

$$
\operatorname{Re} \alpha>\alpha_{c}:=\frac{e^{\omega \beta_{S}}-e^{\omega \beta_{B}}}{\left(e^{\omega \beta_{S}}+1\right)\left(e^{\omega \beta_{B}}-1\right)}>0
$$

for $\beta_{S} / \beta_{B}>1$ (remaining valid for temperatures of arbitrary sign). Conversely, for $\beta_{S} / \beta_{B}<1$, the heat flow reversal happens for

$$
\operatorname{Re} \alpha<\alpha_{c}<0 .
$$

As mentioned in Sec. III, the authorized values of $\operatorname{Re} \alpha$ are limited by the positivity condition of $\rho_{S}^{0}$ which imposes here $|\alpha| \leqslant e^{-\omega \beta_{S}} / Z\left(\beta_{B}\right)$, so that $|\operatorname{Re} \alpha| \leqslant e^{-\omega \beta_{S}} / Z\left(\beta_{B}\right)$. This yields some constraints on the respective values of $\beta_{S}$ and $\beta_{B}$ for which the heat flow can be reversed by internal correlations. For instance, considering a bath at positive temperature, the heat flow can be reversed for $T_{S}<T_{B}$ only if $T_{S}$ is not too small compared to $T_{B}$, in proportions stated by the following inequality:

$$
\omega \beta_{B} \geqslant \omega \beta_{S}-\log \frac{1+2 e^{\omega \beta_{S}}}{2+e^{\omega \beta_{S}}} .
$$

One should note that the term $\log \frac{1+2 e^{\omega \beta_{S}}}{2+e^{\omega \beta_{S}}}$ is always positive, taking values in the interval $[0 ; \log 2]$. Conversely, if $T_{S}>T_{B}>0$, the heat flow can be reversed only if

$$
\omega \beta_{B} \leqslant 2 \omega \beta_{S}+\log \left(1+2 e^{-\omega \beta_{S}}\right) .
$$

Note that (20) is less restrictive than (19). This surprising asymmetry means that it is in general more difficult to increase the apparent temperature from correlations than to reduce it.

\section{A. Illustration}

As illustration of the above ideas, Fig. 1(a) presents plots of $E_{S}=\operatorname{Tr}_{S} \rho_{S} H_{S}$, the energy of $S$, as a function of time (normalized by the characteristic evolution timescale, $G(\omega)^{-1}$; see Appendix C) for values of $\operatorname{Re} \alpha$ ranging from $-e^{-\omega \beta_{S}} / Z\left(\beta_{S}\right)$ to $e^{-\omega \beta_{S}} / Z\left(\beta_{S}\right)$, with $\omega \beta_{S}=3.5$ and $\omega \beta_{B}=4$. Thus, this is a situation where one would expect the heat to flow from $S$ (the hottest) to $B$ (the coldest). The green curve corresponds to no initial correlations whereas the purple curve and the red curves correspond to the minimal and maximal correlations allowed, namely $\operatorname{Re} \alpha=-e^{-\omega \beta_{S}} / Z\left(\beta_{S}\right) \simeq-0.028$ and $\operatorname{Re} \alpha=e^{-\omega \beta_{S}} / Z\left(\beta_{S}\right) \simeq 0.028$, respectively. The blue and orange curves correspond to intermediate values $(\operatorname{Re} \alpha=-0.02$ and $\operatorname{Re} \alpha=0.015$, respectively). The dotted lines associated to each curve indicate the asymptotic value (i.e., the steady-state energy of $S$ ). The derivation of the expression of $E_{S}$ as a function of time is detailed in Appendix C.

These curves deserves several comments. First, one can see from the purple and blue curves that not only is the heat flow reversed for these initial correlations, but also the steady state of the pair has a higher energy than initially (indicated by the dotted colored lines): $S$ is heated up by a colder bath. In the following, we refer to this kind of heat flow reversal between the initial and steady states as permanent heat flow reversal. Note that in principle the conditions (17) and (18) only guarantee the reversal of the heat flow at the initial instant of times, so that the permanent heat flow reversal observed for the purple and blue curves was not necessarily expected. In Appendix D, we expand in more detail on this point and show

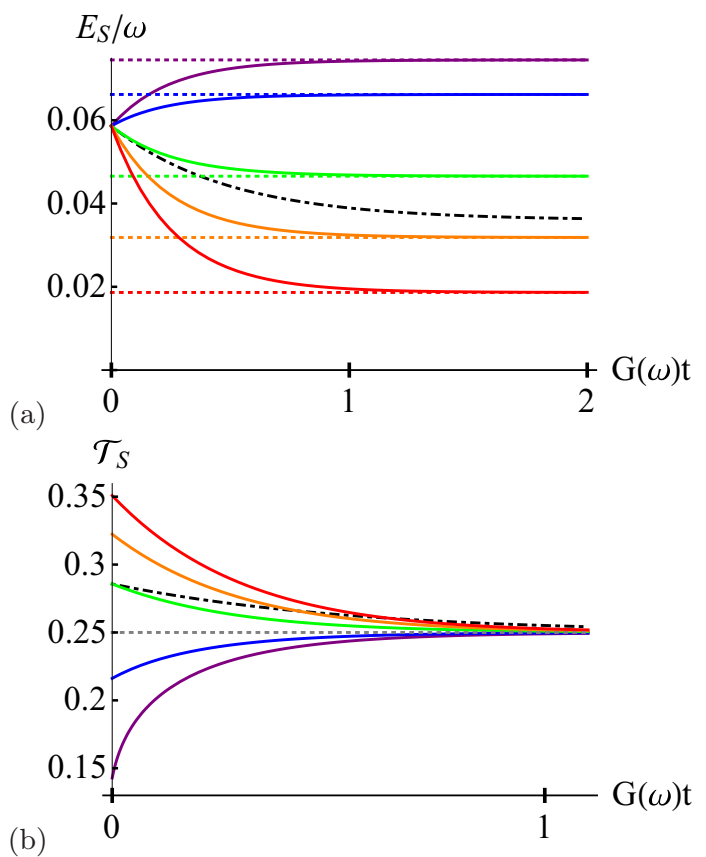

FIG. 1. (a) Plots of the energy $E_{S}$ of $S$ as a function of time (normalized by the characteristic evolution timescale, $G(\omega)^{-1}$; see Appendix C) for different initial correlations and with $\omega \beta_{S}=3.5$ and $\omega \beta_{B}=4$. The purple, blue, green, orange, and red curves corresponds to initial correlations with $\operatorname{Re} \alpha=-e^{-\omega \beta_{S}} / Z\left(\beta_{S}\right) \simeq-0.028$ (the minimal allowed value), $\operatorname{Re} \alpha=-0.02, \operatorname{Re} \alpha=0, \operatorname{Re} \alpha=0.015$, and $\operatorname{Re} \alpha=e^{-\omega \beta_{S}} / Z\left(\beta_{S}\right) \simeq 0,028$ (the maximal allowed value), respectively. The associated dotted lines indicate the asymptotic value of each curve, that is, the steady-state energy of $S$. The dot-dashed black curve is the evolution of the thermal energy (which corresponds to an independent dissipation of each subsystem of $S$ ). (b) Plots of the apparent temperature $\mathcal{T}_{S}$ of $S$ as a function of the (normalized) time for $\omega \beta_{S}=3.5$ and $\omega \beta_{B}=4$. Each curve represents different initial correlations identified by the same color as in panel (a) and the dotdashed black curve represents the time evolution of the temperature for an independent dissipation. The dot gray line represents the bath temperature, to which all apparent temperatures eventually converge.

that the conditions for permanent heat flow reversal are the same as in (9) and (10) when replacing $\alpha_{c}$ with $\alpha_{p}$, where $\left|\alpha_{p}\right|>\left|\alpha_{c}\right|$. In other words, a permanent heat flow reversal requires (slightly) stronger initial correlations than heat flow reversal.

Second, the permanent heat flow reversal can be very important (up to $50 \%$ of the initial energy). This is the object of the next section'. Finally, as a reference, we also plot (dot-dashed black curve) the evolution of the thermal energy which corresponds to the situation where each subsystem interacts independently with $B$ so that it remains in a thermal state at all times and equilibrates to the thermal state at the bath temperature. Comparing the green and dot-dashed black curves, one recovers the effect of mitigation of the bath's action described in Refs. [36,65].

It is also insightful to look at the time evolution of the apparent temperature of $S$. In Fig. 1(b), we plot $\mathcal{T}_{S}$ as a function of (the normalized) time for $\omega \beta_{S}=3.5$ and $\omega \beta_{B}=4$. The derivation of the time evolution of $\mathcal{T}_{S}$ can be found in 


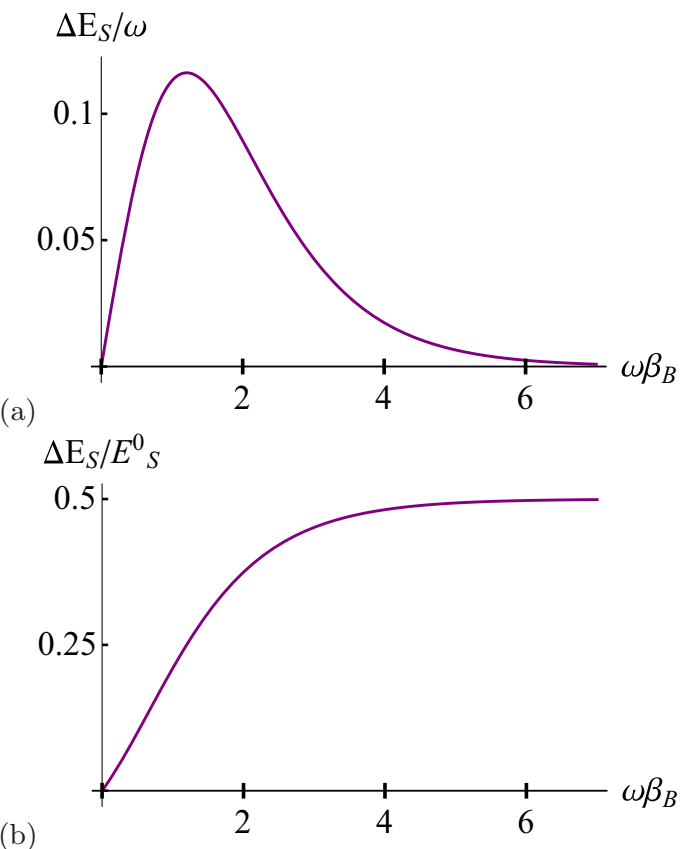

FIG. 2. (a) Plot of the maximal heat flow reversal $\Delta E_{S}:=$ $E_{S}^{\infty}\left(\beta_{B}, \beta_{B}, \operatorname{Re} \alpha_{\min }\right)-E_{S}^{0}\left(\beta_{S}\right)$ as a function of $\omega \beta_{B}$ and for $\beta_{S}=\beta_{B}$. (b) Plot of the maximal heat flow reversal normalized by the initial energy $\Delta E_{S} / E_{S}^{0}\left(\beta_{S}\right)$ as a function of $\omega \beta_{B}$.

Appendix C. Each curve corresponds to a different initial correlation (characterised by $\operatorname{Re} \alpha$ ) identified by the same color as in Fig. 1(a). One can see the huge impact correlations have on the apparent temperature, which explains the subsequent impact on the energy $E_{S}$, Fig. 1(a). Moreover, while reaching the steady state, all curves eventually converge to the bath temperature $1 / \beta_{B}=1 / 4$ (dotted grey line). The dot-dashed black curve represents the time evolution of the temperature for independent dissipation of each subsystems.

Finally, one can also observe that the dissipation process is slower through independent dissipation (dot-dashed black curve) than collective dissipation (full curves). This phenomenon of equilibration speedup stemming from collective dissipation was recently studied in Refs. [66,67].

\section{B. Maximal heat flow reversal}

In this section, we briefly study how large the permanent heat flow reversal can be with respect to the initial energy of $S$. In Appendix C, we derive an expression of the steady-state energy (see also Ref. [36]). We obtain

$\frac{E_{S}^{\infty}\left(\beta_{S}, \beta_{B}, \operatorname{Re} \alpha\right)}{\omega}=1+\frac{\left[\operatorname{Re} \alpha+z\left(\beta_{S}\right)\right]\left(e^{-2 \omega \beta_{B}}-1\right)}{1+e^{-\omega \beta_{B}}+e^{-2 \omega \beta_{B}}}$

with $z\left(\beta_{S}\right):=\left(1+e^{-\omega \beta_{S}}+e^{-2 \omega \beta_{S}}\right) / Z\left(\beta_{S}\right)$, recalling that $Z\left(\beta_{S}\right)=\left(1+e^{-\omega \beta_{S}}\right)^{2}$. For $\beta_{B}>0$ and $\beta_{S}<\beta_{B}$, the maximal heat flow reversal is achieved for an initial correlation with the minimal allowed real part, $\operatorname{Re} \alpha=\operatorname{Re} \alpha_{\min }:=-e^{-\omega \beta_{S}} / Z\left(\beta_{S}\right)$ (implying $\alpha=, \alpha$ ), and for $\beta_{S}$ tending to $\beta_{B}$ (as one could expect). In Fig. 2(a) we show the plot of the maximal heat flow reversal $\Delta E_{S}:=E_{S}^{\infty}\left(\beta_{S}, \beta_{B}, \operatorname{Re} \alpha_{\min }\right)-E_{S}^{0}\left(\beta_{S}\right)$ as a function of $\omega \beta_{B}$ for $\beta_{S}=\beta_{B}$, where $E_{S}^{0}\left(\beta_{S}\right)$ denotes the initial energy, equal to the thermal energy at inverse temperature $\beta_{S}$, namely $E_{S}^{0}\left(\beta_{S}\right)=2 \frac{e^{-\omega \beta_{S}}}{1+e^{-\omega \beta_{S}}}$. One can see that the permanent heat flow reversal can go up to an energy equal to $0.12 \omega$ and represent a gain of energy up to $50 \%$ of the initial energy, as shown in Fig. 2(b). This is a very significant effect.

For $\beta_{S}>\beta_{B}>0$, the maximal heat flow reversal is achieved for an initial correlation with the maximal allowed real part, $\operatorname{Re} \alpha=\operatorname{Re} \alpha_{\max }:=e^{-\omega \beta_{S}} / Z\left(\beta_{S}\right)$ (implying $\alpha=\operatorname{Re} \alpha$ ) and for $\beta_{S}$ tending to $\beta_{B}$. The corresponding plots are the same as in Figs. 2(a) and 2(b) but with negative signs. Finally, for $\beta_{B}<0$, the results are similar (and can be obtained from the above ones just by inverting $\operatorname{Re} \alpha_{\min }$ and $\left.\operatorname{Re} \alpha_{\max }\right)$.

\section{Conversion of correlations into energy}

In the previous section, Sec. VB, we show that maximal (permanent) heat flow reversals happen for extremal values of the initial correlations and for $\beta_{S}=\beta_{B}$. This situation is also interesting by itself: The ongoing energy exchanges occur only thanks to the initial correlations. In other words, this is a direct conversion of correlations into energy. Driven by curiosity about this intriguing process, we mention some of its interesting properties. The following considerations go slightly beyond the scope of this paper, but we found them worth the following brief overview.

We first focus on the variation of entropy of $S$ during the conversion process. Using the expression of the steady state derived in Appendix $\mathrm{C}$, one can derive the expression of $S_{S}^{\infty}$, the steady-state entropy of $S$ (see Appendix E for the detail of the expression). Figure 3(a) displays the plot of the the variation of entropy $\Delta S_{S}:=S_{S}^{\infty}-S_{S}^{0}$ between the initial and steady states as a function of $\omega \beta_{B}$ for $\alpha=\operatorname{Re} \alpha=\operatorname{Re} \alpha_{\min }$

(a)

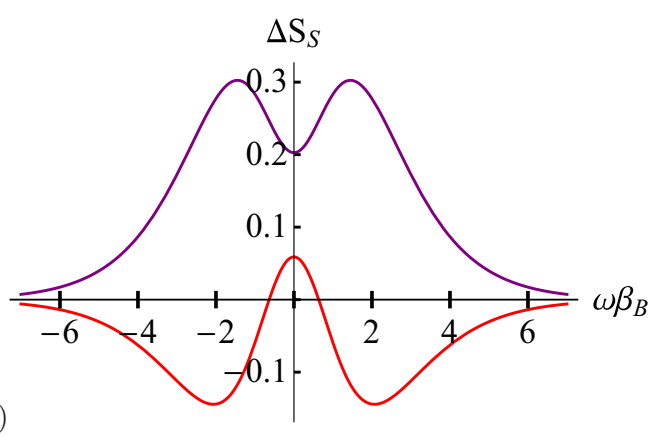

$\Delta \mathrm{E}_{S} / \omega$

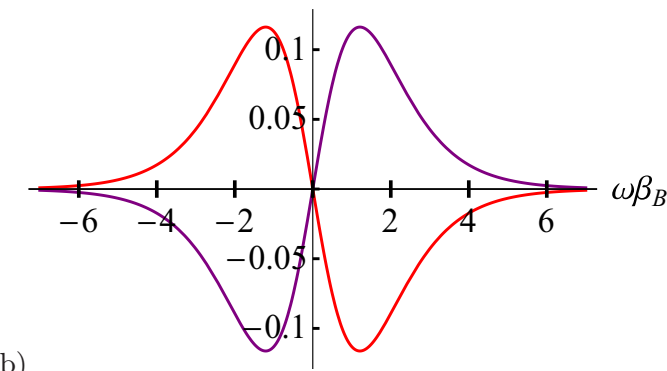

(b)

FIG. 3. Plots of (a) the entropy variation $\Delta S_{S}=S_{S}^{\infty}-S_{S}^{0}$ between the initial and steady states of $S$ and (b) the energy exchange $\Delta E_{S}=E_{S}^{\infty}-E_{S}^{0}$, as functions of $\omega \beta_{B}$ for $\operatorname{Re} \alpha=\operatorname{Re} \alpha_{\min }$ (purple curves) and $\operatorname{Re} \alpha=\operatorname{Re} \alpha_{\max }$ (red curves). 
(purple curve) and $\alpha=\operatorname{Re} \alpha=\operatorname{Re} \alpha_{\max }$ (red curve). It is insightful to analyze these curves while keeping an eye on Fig. 3(b), which displays the energy exchange $\Delta E_{S}$ as a function of $\omega \beta_{B}$, still for $\alpha=\operatorname{Re} \alpha=\operatorname{Re} \alpha_{\min }$ (purple curve) and $\alpha=\operatorname{Re} \alpha=\operatorname{Re} \alpha_{\max }$ (red curve). Note that Fig. 3(b) is just an extension of Fig. 2(a) to negative bath temperature and displays the symmetric situation of $\alpha=\operatorname{Re} \alpha=\operatorname{Re} \alpha_{\max }$.

Interestingly, one can see that the value $\omega \beta_{B} \simeq 0.7$ is very peculiar for the red curve. Indeed, around this point, the initial and final entropies are the same while the energy variation is highly negative, as shown by Fig. 3(b). We have here an interesting setup which reproduces perfectly (at least around the value $\omega \beta_{B}=0.7$ ) the ideal quantum battery or external (classical) energy source [37,38]: It delivers energy without changing its entropy, which corresponds to the idea of perfect work generator. Then, could one say that this process is a conversion of correlations into perfect work? It also rises recurrent questions regarding the nature of the difference between heat and work as it is usually understood that energy exchanges with a thermal bath are exclusively heat (independently of the sign of the energy exchanges).

\section{CONCLUSION}

This work presents a surprising phenomenon: Indistinguishability and correlations between subsystems can reverse the heat flow between an ensemble and its bath. Additionally, in contrast to previous works $[1-3,5,6]$, this does not involve reversal of the arrow of time. This phenomenon is first unveiled in a broader context, including several types of degenerate systems, as a consequence of the impact of correlations and nonenergetic coherences on the system's apparent temperature. This emphasizes that the apparent temperature [35] is not merely a mathematical tool but that it has real physical meaning and consequences which could be tested experimentally relatively easily in pairs of two-level systems.

Then, adopting the formalism used in Refs. [1,6], we provide an alternative view: The degradation of initial internal correlations provides a kind of "fuel" for heat flow reversal. Still, there is a fundamental difference with Refs. [1,6]: In our protocol, system and bath are initially uncorrelated, which guarantees the positivity of the entropy production and therefore does not affect the arrow of time. However, we show that initial internal correlations (correlations within $S$ ) can also lead to heat flow reversal. In this sense, our setup has the advantage that it does not need previous preparation steps involving both system and bath (not always accessible or controllable), but only preparation steps on the system. Note also that since the reported effect only requires a degenerate system or correlated subsystems interacting collectively with a bath, it might occur in several many-body experimental setups or even in organic structures.

Aiming at accessible experimental realisations, we then focus on a pair of two-level systems (used in Ref. [1]). We show that the heat flow reversal can be very large, leading to permanent heat flow reversal of up to $50 \%$ of the initial energy. One might expect this phenomenon to become larger as the number of subsystems increases in a similar way as indistinguishability has growing impact in spin ensembles of increasing spin number [65]. It would be indeed interesting to investigate such heat flow reversal in larger systems.
We finally broaden the study, pointing at intriguing processes of correlations-to-energy conversion. In particular, we point out a regime where the correlations-to-energy conversion operates at constant entropy, reproducing the behavior of the external energy source or pure work source.

Our results uncover the central role played by nonenergetic quantum coherences in collective dissipative processes, leading to diverse curious phenomena such as heat flow reversals. This suggests that the heat flow reversal pointed out in this paper has no classical counterpart unlike the one previously known relying on initial correlations between system and bath. An interesting topic would be to investigate in more detail whether nonenergetic coherences also play a special role in entropy production as compared to the role played by energetic coherences recently studied in Ref. [68].

\section{ACKNOWLEDGMENTS}

This work is based upon research supported by the South African Research Chair Initiative, Grant No. UID 64812 of the Department of Science and Technology of the Republic of South Africa and National Research Foundation of the Republic of South Africa.

\section{APPENDIX A: HEAT FLOW AND APPARENT TEMPERATURE}

The reduced dynamics of $S$ is given by the following Markovian master equation (valid under weak coupling):

$$
\begin{aligned}
\dot{\rho}_{t}= & \Gamma(\omega)\left[\mathcal{A} \rho_{t} \mathcal{A}^{\dagger}-\mathcal{A}^{\dagger} \mathcal{A} \rho_{t}\right]+\text { H.c. } \\
& +\Gamma(-\omega)\left[\mathcal{A}^{\dagger} \rho_{t} \mathcal{A}-\mathcal{A} \mathcal{A}^{\dagger} \rho_{t}\right]+\text { H.c. },
\end{aligned}
$$

where $\Gamma(\omega)=\int_{0}^{\infty} d s e^{i \omega s} \operatorname{Tr} \rho_{B} B(s) B$, and $B(s)$ is the bath operator $B$ introduced in (1) in the interaction picture (with respect to its free Hamiltonian). The heat flow between $S$ and $B$, given by the energy variation $E_{S}^{t}:=\operatorname{Tr} \dot{\rho}_{S}^{t} H_{S}$, can be expressed using the above master equation. Using the commutation relation of the eigenoperator $\left[\mathcal{A}^{\dagger}, H_{S}\right]=-\omega \mathcal{A}^{\dagger}$, one can rewrite $\dot{E}_{S}$ in the following interesting form,

$$
\dot{E}_{S}=\omega G(\omega)\left\langle\mathcal{A} \mathcal{A}^{\dagger}\right\rangle_{\rho_{S}^{t}}\left(e^{-\omega / T_{B}}-e^{-\omega / \mathcal{T}_{S}}\right),
$$

where $G(\omega):=\Gamma(\omega)+\Gamma^{*}(\omega)=\int_{-\infty}^{\infty} d s e^{i \omega s} \operatorname{Tr} \rho_{B} B(s) B$ and the bath temperature $T_{B}$ can be defined through $e^{-\omega / T_{B}}:=$ $G(-\omega) / G(\omega)[35,69,70]$. The apparent temperature [35] $\mathcal{T}_{S}:=\omega\left(\ln \frac{\left\langle\mathcal{A} \mathcal{A}^{\dagger}\right\rangle_{\rho_{S}}}{\left\langle\mathcal{A}^{\dagger} \mathcal{A}\right\rangle_{\rho_{S}}}\right)^{-1}$, introduced in the main text in (4), determines the sign of the heat flow between $S$ and $B$. Indeed, $\dot{E}_{S} \geqslant 0\left(\dot{E}_{S} \leqslant 0\right)$ if and only if $\mathcal{T}_{S} \leqslant 1 / \beta_{B}\left(\mathcal{T}_{S} \geqslant 1 / \beta_{B}\right)$. Thus, $\mathcal{T}_{S}$ can be thought as an apparent temperature of $S$, seen from the point of view of $B$, extending the notion of temperature to nonthermal states. Importantly, when $S$ is in a thermal state, the apparent temperature coincides with the usual notion of temperature. One interesting property of the apparent temperature is to take into account contributions from coherences and correlations [35].

\section{APPENDIX B: EQUALITY OF $\left\langle\mathcal{A}^{\dagger} \mathcal{A}\right\rangle_{\mathrm{cor}}=\left\langle\mathcal{A} \mathcal{A}^{\dagger}\right\rangle_{\mathrm{cor}}$}

The equality of $\left\langle\mathcal{A}^{\dagger} \mathcal{A}\right\rangle_{\text {cor }}=\left\langle\mathcal{A} \mathcal{A}^{\dagger}\right\rangle_{\text {cor }}$ mentioned in the main text is a direct consequence of the delocalized nature of the correlation term $\chi^{0}$. Denoting by $a_{i}$ and $a_{i}^{\dagger}$ the local 
eigenoperators of each subsystems $S_{i}$ so that $\mathcal{A}=\sum_{i} a_{i}$ $\left(\mathcal{A}^{\dagger}=\sum_{i} a_{i}^{\dagger}\right)$, we have

$$
\begin{aligned}
\left\langle\mathcal{A}^{\dagger} \mathcal{A}\right\rangle_{\text {cor }} & =\sum_{i, j} \operatorname{Tr} \chi^{0} a_{i} a_{j}^{\dagger} \\
& =\sum_{i} \operatorname{Tr} \chi^{0} a_{i} a_{i}^{\dagger}+\sum_{i \neq j} \operatorname{Tr} \chi^{0} a_{i} a_{j}^{\dagger} \\
& =\sum_{i \neq j} \operatorname{Tr} \chi^{0} a_{i} a_{j}^{\dagger} \\
& =\sum_{i \neq j} \operatorname{Tr} \chi^{0} a_{j}^{\dagger} a_{i} \\
& =\operatorname{Tr} \chi^{0} \mathcal{A}^{\dagger} \mathcal{A} \\
& =\left\langle\mathcal{A} \mathcal{A}^{\dagger}\right\rangle_{\mathrm{cor}},
\end{aligned}
$$

where the term $\operatorname{Tr} \chi^{0} a_{i} a_{i}^{\dagger}=\operatorname{Tr} \chi^{0} a_{i}^{\dagger} a_{i}=\operatorname{Tr}_{i} a_{i}^{\dagger} a_{i} \operatorname{Tr}_{S / S_{i}}\left(\chi^{0}\right)$ is null for all $i$ since $\operatorname{Tr}_{S / S_{i}}\left(\chi^{0}\right)$, the partial trace of $\chi^{0}$ over all subsystems other than $S_{i}$, is null (the correlations do not contribute to the local states).

\section{APPENDIX C: EXPRESSION OF THE ENERGY OF $S$ AS A FUNCTION OF TIME}

The following derivation was already detailed in the Supplementary Material of Ref. [35] (Sec. VIII). We reproduce it here for the sake of completeness. Based on the form of the system-bath coupling (1), one can derive the following master equation, valid under weak coupling, which legitimates the Born and Markov approximations [56,57],

$$
\begin{aligned}
\dot{\rho}_{S}^{I}= & -i \Omega_{L} \sum_{i=1}^{2}\left[\sigma_{i}^{+} \sigma_{i}^{-}, \rho_{S}^{I}\right]-i \Omega_{1,2}\left[\sigma_{1}^{+} \sigma_{2}^{-}+\sigma_{1}^{-} \sigma_{2}^{+}, \rho_{S}^{I}\right] \\
& +G(\omega)\left(2 S^{-} \rho_{S}^{I} S^{+}-S^{+} S^{-} \rho_{S}^{I}-\rho_{S}^{I} S^{+} S^{-}\right) \\
& +G(-\omega)\left(2 S^{+} \rho_{S}^{I} S^{-}-S^{-} S^{+} \rho_{S}^{I}-\rho_{S}^{I} S^{-} S^{+}\right) .
\end{aligned}
$$

In the above equation, we used the bath spectral density $G(\omega)$ introduced in Appendix A. Furthermore, the operators $\sigma_{i}^{+}$and $\sigma_{i}^{-}$are the ladder operators of the two-level system $S_{i}$ (spin or atom) defined in Sec. V, $S^{+}=\sum_{i=1}^{2} \sigma_{i}^{+}$and $S^{-}=\sum_{i=1}^{2} \sigma_{i}^{-}$ are the collective ladder operators also introduced in Sec. V, $\Omega_{L}$ is the Lamb shift, and the term proportional to $\Omega_{1,2}$ corresponds to the interaction between the two subsystems $S_{1}$ and $S_{2}$ (which can be taken to zero if the two subsystems do not interact). The above master equation has the same form as the one describing the dynamics of a pair of two-level atoms interacting with the free space electromagnetic field [42]. In such a case, the interaction $\Omega_{1,2}$ corresponds to the van der Waals interaction. Note also that the above master equation is valid for any stationary bath $[35,69,70]$, which includes some nonthermal baths. In such situations, the bath inverse temperature $\beta_{B}$ is substituted by the inverse of the bath apparent temperature which can be simply defined as $\omega[\log [G(\omega) / G(-\omega)]]^{-1}[35,69]$.

The dynamics described by $(\mathrm{C} 1)$ can be easily solved by considering the basis $\left\{\left|\psi_{0}\right\rangle,\left|\psi_{+}\right\rangle,\left|\psi_{-}\right\rangle,\left|\psi_{1}\right\rangle\right\}$ with $\left|\psi_{ \pm}\right\rangle=$ $(|01\rangle \pm|10\rangle) / \sqrt{2},\left|\psi_{0}\right\rangle=|00\rangle$, and $\left|\psi_{1}\right\rangle=|11\rangle$. In such basis, the collective ladder operators can be expressed as
$S^{+}=\sqrt{2}\left|\psi_{+}\right\rangle\left\langle\psi_{0}|+\sqrt{2}| \psi_{1}\right\rangle\left\langle\psi_{+}\right|$and $S^{-}=\sqrt{2}\left|\psi_{0}\right\rangle\left\langle\psi_{+}\right|+$ $\sqrt{2}\left|\psi_{+}\right\rangle\left\langle\psi_{1}\right|$. From (C1), one obtains the following dynamics for the populations $p_{i}:=\left\langle\psi_{i}\left|\rho_{S}\right| \psi_{i}\right\rangle, i=0,1,+,-$,

$$
\begin{aligned}
& \dot{p}_{1}=4 G(-\omega) p_{+}-4 G(\omega) p_{1}, \\
& \dot{p}_{0}=4 G(\omega) p_{+}-4 G(-\omega) p_{0}, \\
& \dot{p}_{+}=4 G(\omega)\left(p_{1}-p_{+}\right)+4 G(-\omega)\left(p_{0}-p_{+}\right), \\
& \dot{p}_{-}=0 .
\end{aligned}
$$

The steady-state populations can be obtained by canceling the time derivatives in the above system of equations. Alternatively, one can also solve the above system to obtain the time evolution of the populations. This is simplified by noting that $\dot{p}_{1}+\dot{p}_{0}+\dot{p}_{+}=0$, which implies that $r:=p_{1}+p_{0}+p_{+}$is a constant determined by the initial conditions. The system can therefore be reduced to a system of two linearly independent equations (substituting, for instance, $p_{1}$ by $r-p_{0}-p_{+}$),

$$
\begin{aligned}
\dot{p}_{0}= & 4 G(\omega) p_{+}-4 G(-\omega) p_{0}, \\
\dot{p}_{+}= & -4[G(\omega)-G(-\omega)] p_{0}-4[2 G(\omega)+G(-\omega)] p_{+} \\
& +4 G(\omega) r .
\end{aligned}
$$

The reduced system is diagonalized by the quantities $q^{ \pm}:=$ $p_{+}+[1 \pm \sqrt{G(-\omega) / G(\omega)}] p_{0}$, with the associated eigenvalues $a^{ \pm}:=4[ \pm \sqrt{G(\omega) G(-\omega)}-G(\omega)-G(-\omega)]$, so that

$$
\dot{q}^{ \pm}=a^{ \pm} q^{ \pm}+4 G(\omega) r
$$

and

$$
q^{ \pm}(t)=e^{a^{ \pm} t} q^{ \pm}(0)+4 G(\omega) r \frac{a^{a^{ \pm} t}-1}{a^{ \pm}} .
$$

From the time evolution of $q^{ \pm}(t)$, one can deduce straightforwardly the expression for the time evolution of the populations $p_{0}, p_{+}, p_{1}$, the energy $E_{S} / \omega=2 p_{1}+p_{+}+p_{-}$, and the apparent temperature $\mathcal{T}_{S}=\omega\left(\log \frac{p_{0}+p_{+}}{p_{1}+p_{+}}\right)^{-1}$. Applying the deduced expressions for different values of the initial correlations ( $\operatorname{Re} \alpha$ varying from the minimal to the maximal allowed value), one obtains the plots of Fig. 1.

The corresponding steady-state populations are

$$
\begin{aligned}
& p_{0}^{\infty}=r \frac{1}{1+e^{-\omega \beta_{B}}+e^{-2 \omega \beta_{B}}}, \\
& p_{+}^{\infty}=r \frac{e^{-\omega \beta_{B}}}{1+e^{-\omega \beta_{B}}+e^{-2 \omega \beta_{B}}}, \\
& p_{1}^{\infty}=r \frac{e^{-2 \omega \beta_{B}}}{1+e^{-\omega \beta_{B}}+e^{-2 \omega \beta_{B}}}, \\
& p_{-}^{\infty}=1-r,
\end{aligned}
$$

which yields a steady-state energy equal to

$$
E_{S}^{\infty}=1+r \frac{e^{-2 \omega \beta_{B}}-1}{1+e^{-\omega \beta_{B}}+e^{-2 \omega \beta_{B}}} .
$$

When the initial state is of the form (7), the constant $r$ is equal to

$$
r=\operatorname{Re} \alpha+z\left(\beta_{S}\right),
$$

where $z\left(\beta_{S}\right):=\left(1+e^{-\omega \beta_{S}}+e^{-2 \omega \beta_{S}}\right) / Z\left(\beta_{S}\right)$, which leads for the steady-state energy to the expression (21) announced in the main text. 
For the coherences, defined as $\rho_{i j}:=\left\langle\psi_{i}\left|\rho_{S}^{I}\right| \psi_{j}\right\rangle, i, j \in$ $\{0,1,+,-\}$, one obtains (including the Lamb shift in the interaction picture)

$$
\begin{aligned}
\dot{\rho}_{+,-} & =-2\left[G(\omega)+G(-\omega)+i \Omega_{I}\right] \rho_{+,-}, \\
\dot{\rho}_{1,-} & =-\left[2 G(\omega)+i \Omega_{I}\right] \rho_{1,-}, \\
\dot{\rho}_{0,-} & =-\left[2 G(-\omega)+i \Omega_{I}\right] \rho_{0,-}, \\
\dot{\rho}_{1,0} & =-2[G(\omega)+G(-\omega)] \rho_{1,0},
\end{aligned}
$$

which straightforwardly give 0 as the steady-state solution. The dynamics of the two remaining coherences is coupled,

$$
\begin{aligned}
& \dot{\rho}_{1,+}=-\left\{2[2 G(\omega)+G(-\omega)]-i \Omega_{I}\right\} \rho_{1,+}+4 G(-\omega) \rho_{+, 0}, \\
& \dot{\rho}_{+, 0}=-\left\{2 g[G(\omega)+2 G(-\omega)]+i \Omega_{I}\right\} \rho_{+, 0}+4 G(\omega) \rho_{1,+},
\end{aligned}
$$

and also leads to 0 as a steady-state solution. Finally, one can write the steady state in the form

$$
\begin{aligned}
\rho_{S}^{\infty}= & \frac{r}{1+e^{-\omega \beta_{B}}+e^{-2 \omega \beta_{B}}}\left(e^{-2 \omega \beta_{B}}\left|\psi_{1}\right\rangle\left\langle\psi_{1}\left|+e^{-\omega \beta_{B}}\right| \psi_{+}\right\rangle\left\langle\psi_{+}\right|\right. \\
& \left.+\left|\psi_{0}\right\rangle\left\langle\psi_{0}\right|\right)+(1-r)\left|\psi_{-}\right\rangle\left\langle\psi_{-}\right| .
\end{aligned}
$$

\section{APPENDIX D: PERMANENT HEAT FLOW REVERSAL}

As commented in Sec. V in the main text, the conditions (9) and (10) correspond to reversal of the heat flow at initial times but do not guarantee that the heat flow remains inverted throughout times until $S$ reaches its steady state, what we call permanent reversal of the heat flow. We show now that a permanent reversal requires indeed a higher level of initial correlations. From the expression of the steady-state energy (C7) (or (21) in the main text)

$$
E_{S}^{\infty}=1+\frac{\left[\operatorname{Re} \alpha+z\left(\beta_{S}\right)\right]\left(e^{-2 \omega \beta}-1\right)}{1+e^{-\omega \beta_{B}}+e^{-2 \omega \beta_{B}}},
$$

compared to the initial energy $E_{S}^{0}:=\operatorname{Tr} \rho_{S}^{0} H_{S}=2 \frac{e^{-\omega \beta_{S}}}{\left(1+e^{\omega \beta_{S}}\right.}$, one obtains that a permanent heat flow reversal takes place if and only if

$$
\begin{array}{ll}
\operatorname{Re} \alpha>\alpha_{p}>0, & \text { for } \beta_{\mathrm{S}} / \beta_{\mathrm{B}}>1, \\
\operatorname{Re} \alpha<\alpha_{p}<0, & \text { for } \beta_{\mathrm{S}} / \beta_{\mathrm{B}}<1,
\end{array}
$$

with $\alpha_{p}:=z\left(\beta_{B}\right) \frac{1+e^{-\omega \beta_{B}}}{1-e^{-\omega \beta_{B}}} \frac{1-e^{-\omega \beta_{S}}}{1+e^{-\omega \beta_{S}}}-z\left(\beta_{S}\right)$. Note that the above conditions are valid for $\beta_{S}$ and $\beta_{B}$ of arbitrary sign. A comparison with $\alpha_{c}$ the critical value required for $\operatorname{Re} \alpha$ to induce heat flow reversal introduced in (9) and (10) shows that $\left|\alpha_{c}\right|<$ $\left|\alpha_{p}\right|$, which implies that permanent heat flow reversal always requires a higher level of correlations and coherences.

\section{APPENDIX E: EXPRESSION OF THE STEADY STATE ENTROPY}

The steady-state entropy (von Neumann entropy) can be obtained from the expression of the steady state (C11). Since the expression is given in a basis that diagonalizes $\rho_{S}^{\infty}$, it is straightforward to show that the steady-state entropy is

$$
\begin{aligned}
S_{S}^{\infty}:= & -\operatorname{Tr} \rho_{S}^{\infty} \ln \rho_{S}^{\infty} \\
= & r \ln \left[\left(1+e^{-\omega \beta_{B}}+e^{-2 \omega \beta_{B}}\right) / r\right]-(1-r) \ln (1-r) \\
& +r \omega \beta_{B} \frac{e^{-\omega \beta_{B}}+e^{-2 \omega \beta_{B}}}{1+e^{-\omega \beta_{B}}+e^{-2 \omega \beta_{B}}},
\end{aligned}
$$

where $\quad r:=\left\langle\psi_{0}\left|\rho_{S}^{0}\right| \psi_{S}\right\rangle+\left\langle\psi_{0}\left|\rho_{S}^{0}\right| \psi_{0}\right\rangle+\left\langle\psi_{S}^{0}\left|\rho_{0}\right| \psi_{0}\right\rangle \quad$ is a constant introduced in Appendix $\mathrm{C}$ together with the basis $\left\{\left|\psi_{0}\right\rangle,\left|\psi_{+}\right\rangle,\left|\psi_{-}\right\rangle,\left|\psi_{1}\right\rangle\right\}$ defined by $\left|\psi_{ \pm}\right\rangle=$ $(|01\rangle \pm|10\rangle) / \sqrt{2},\left|\psi_{0}\right\rangle=|00\rangle$, and $\left|\psi_{1}\right\rangle=|11\rangle$. For initial states of the form (7) with $\chi^{0}$ given by (16), one has simply $r=z\left(\beta_{S}\right)+\operatorname{Re} \alpha$, with $z\left(\beta_{S}\right):=\left(1+e^{-\omega \beta_{S}}+e^{-2 \omega \beta_{S}}\right) / Z\left(\beta_{S}\right)$. Such initial states can be rewritten in a diagonal form as

$$
\begin{aligned}
\rho_{S}^{0}= & \frac{e^{-2 \omega \beta_{B}}}{Z\left(\beta_{B}\right)}\left|\psi_{1}\right\rangle\left\langle\psi_{1}\left|+\left[\frac{e^{-\omega \beta_{B}}}{Z\left(\beta_{B}\right)}+|\alpha|\right]\right| \psi_{+, \phi}\right\rangle\left\langle\psi_{+, \phi}\right| \\
& +\left[\frac{e^{-\omega \beta_{B}}}{Z\left(\beta_{B}\right)}-|\alpha|\right]\left|\psi_{-, \phi}\right\rangle\left\langle\psi_{-, \phi}\left|+\frac{1}{Z\left(\beta_{B}\right)}\right| \psi_{0}\right\rangle\left\langle\psi_{0}\right|
\end{aligned}
$$

in the basis $\left\{\left|\psi_{0}\right\rangle,\left|\psi_{+}\right\rangle,\left|\psi_{-}\right\rangle,\left|\psi_{1}\right\rangle\right\}$ defined by $\left|\psi_{ \pm, \phi}\right\rangle=$ $\left(e^{i \phi}|01\rangle \pm e^{-i \phi}|10\rangle\right) / \sqrt{2},\left|\psi_{0}\right\rangle=|00\rangle,\left|\psi_{1}\right\rangle=|11\rangle$, and the phase $\phi:=\arg \alpha$ is the argument of the correlations. Then, the initial entropy can be obtained straightforwardly:

$$
\begin{aligned}
S_{S}^{0}:= & -\operatorname{Tr} \rho_{S}^{0} \ln \rho_{s}^{0} \\
= & \ln Z\left(\beta_{S}\right)+2 \omega \beta_{S} \frac{e^{-\omega \beta_{S}}}{1+e^{-\omega \beta_{S}}} \\
& -\left[\frac{e^{-\omega \beta_{S}}}{Z\left(\beta_{S}\right)}+|\alpha|\right] \ln \left[1+|\alpha| e^{\omega \beta_{S}} Z\left(\beta_{S}\right)\right] \\
& -\left[\frac{e^{-\omega \beta_{S}}}{Z\left(\beta_{S}\right)}-|\alpha|\right] \ln \left[1-|\alpha| e^{\omega \beta_{S}} Z\left(\beta_{S}\right)\right] .
\end{aligned}
$$

By combining (E2) and (E3), one can obtain an expression of the variation of entropy $\Delta S_{S}:=S_{S}^{\infty}-S_{S}^{0}$, which was used to plot the graph in Fig. 3(a).
[1] K. Micadei, J. P. S. Peterson, A. M. Souza, R. S. Sarthour, I. S. Oliveira, G. T. Landi, T. B. Batalhão, R. M. Serra, and E. Lutz, Nat. Commun. 10, 2456 (2019).

[2] M. H. Partovi, Phys. Rev. E 77, 021110 (2008).

[3] S. Jevtic, D. Jennings, and T. Rudolph, Phys. Rev. Lett. 108, 110403 (2012).

[4] S. Jevtic, T. Rudolph, D. Jennings, Y. Hirono, S. Nakayama, and M. Murao, Phys. Rev. E 92, 042113 (2015).

[5] I. Henao and R. M. Serra, Phys. Rev. E 97, 062105 (2018).

[6] D. Jennings and T. Rudolph, Phys. Rev. E 81, 061130 (2010).
[7] A. Levi and M. Lostaglio, arXiv:1909.11116.

[8] J. Oppenheim, M. Horodecki, P. Horodecki, and R. Horodecki, Phys. Rev. Lett. 89, 180402 (2002).

[9] M. O. Scully, M. S. Zubairy, G. S. Agarwal, and H. Walther, Science 299, 862 (2003).

[10] K. V. Hovhannisyan, M. Perarnau-Llobet, M. Huber, and A. Acín, Phys. Rev. Lett. 111, 240401 (2013).

[11] M. Perarnau-Llobet, K. V. Hovhannisyan, M. Huber, P. Skrzypczyk, N. Brunner, and A. Acín, Phys. Rev. X 5, 041011 (2015). 
[12] J. Åberg, Phys. Rev. Lett. 113, 150402 (2014).

[13] R. Dillenschneider and E. Lutz, Europhys. Lett. 88, 50003 (2009).

[14] M. O. Scully, K. R. Chapin, K. E. Dorfman, M. B. Kim, and A. Svidzinsky, Proc. Natl. Acad. Sci. USA 108, 15097 (2011).

[15] R. Uzdin, A. Levy, and R. Kosloff, Phys. Rev. X 5, 031044 (2015).

[16] W. Niedenzu, D. Gelbwaser-Klimovsky, and G. Kurizki, Phys. Rev. E 92, 042123 (2015).

[17] F. Altintas, A. Ü. C. Hardal, and Ö. E. Müstecaplıolu, Phys. Rev. A 91, 023816 (2015).

[18] R. Uzdin, Phys. Rev. Appl. 6, 024004 (2016).

[19] H.-B. Chen, P.-Y. Chiu, and Y.-N. Chen, Phys. Rev. E 94, 052101 (2016).

[20] G. A. Barrios, F. Albarrán-Arriagada, F. A. Cárdenas-López, G. Romero, and J. C. Retamal, Phys. Rev. A 96, 052119 (2017).

[21] F. C. Binder, S. Vinjanampathy, K. Modi, and J. Goold, New J. Phys. 17, 075015 (2015).

[22] D. Ferraro, M. Campisi, G. M. Andolina, V. Pellegrini, and M. Polini, Phys. Rev. Lett. 120, 117702 (2018).

[23] P. Rebentrost, M. Mohseni, I. Kassal, S. Lloyd, and A. AspuruGuzik, New J. Phys. 11, 033003 (2009).

[24] F. Caruso, A. W. Chin, A. Datta, S. F. Huelga, and M. B. Plenio, J. Chem. Phys. 131, 105106 (2009).

[25] A. Ishizaki and G. R. Fleming, J. Chem. Phys. 130, 234111 (2009).

[26] S. Lloyd, J. Phys.: Conf. Ser. 302, 012037 (2011).

[27] M. H. Lee and A. Troisi, J. Chem. Phys. 146, 075101 (2017).

[28] M. O. Scully, Phys. Rev. Lett. 104, 207701 (2010).

[29] A. A. Svidzinsky, K. E. Dorfman, and M. O. Scully, Phys. Rev. A 84, 053818 (2011).

[30] C. Creatore, M. A. Parker, S. Emmott, and A. W. Chin, Phys. Rev. Lett. 111, 253601 (2013).

[31] E. Romero, R. Augulis, V. I. Novoderezhkin, M. Ferretti, J. Thieme, D. Zigmantas, and R. van Grondelle, Nat. Phys. 10, 676 (2014).

[32] E. Collini, C. Y. Wong, K. E. Wilk, P. M. G. Curmi, P. Brumer, and G. D. Scholes, Nature (London) 463, 644 (2010).

[33] S. F. Huelga and M. B. Plenio, Contemp. Phys. 54, 181 (2013).

[34] A. W. Chin, J. Prior, R. Rosenbach, F. Caycedo-Soler, S. F. Huelga, and M. B. Plenio, Nat. Phys. 9, 113 (2013).

[35] C. L. Latune, I. Sinayskiy, and F. Petruccione, Quantum Sci. Technol. 4, 025005 (2019).

[36] C. L. Latune, I. Sinayskiy, and F. Petruccione, Phys. Rev. A 99, 052105 (2019).

[37] R. Kosloff, Entropy 15, 2100 (2013).

[38] R. Kosloff and A. Levi, Annu. Rev. Phys. Chem. 65, 365 (2014).

[39] A. Rivas and M. A. Martin-Delgado, Sci. Rep. 7, 6350 (2017).

[40] M. O. Scully and M. S. Zubairy, Quantum Optics (Cambridge University Press, London, 1997).

[41] T. V. Tscherbul and P. Brumer, J. Chem. Phys. 142, 104107 (2015).

[42] M. Gross and S. Haroche, Phys. Rep. 93, 301 (1982).

[43] R. G. DeVoe and R. G. Brewer, Phys. Rev. Lett. 76, 2049 (1996).
[44] M. D. Barnes, P. S. Krstic, P. Kumar, A. Mehta, and J. C. Wells, Phys. Rev. B 71, 241303(R) (2005).

[45] C. J. Wood, T. W. Borneman, and D. G. Cory, Phys. Rev. Lett. 112, 050501 (2014).

[46] C. J. Wood and D. G. Cory, Phys. Rev. A 93, 023414 (2016).

[47] Y. Hama, W. J. Munro, and K. Nemoto, Phys. Rev. Lett. 120, 060403 (2018).

[48] W. Niedenzu and G. Kurizki, New J. Phys. 20, 113038 (2018).

[49] J. M. Raimond, P. Goy, M. Gross, C. Fabre, and S. Haroche, Phys. Rev. Lett. 49, 117 (1982).

[50] L. Moi, P. Goy, M. Gross, J. M. Raimond, C. Fabre, and S. Haroche, Phys. Rev. A 27, 2043 (1983).

[51] A. Goban, C.-L. Hung, J. D. Hood, S.-P. Yu, J. A. Muniz, O. Painter, and H. J. Kimble, Phys. Rev. Lett. 115, 063601 (2015).

[52] W. Guerin, M. O. Araújo, and R. Kaiser, Phys. Rev. Lett. 116, 083601 (2016).

[53] M. O. Araújo, I. Krešić, R. Kaiser, and W. Guerin, Phys. Rev. Lett. 117, 073002 (2016).

[54] D. Barberena, R. J. Lewis-Swan, J. K. Thompson, and A. M. Rey, Phys. Rev. A 99, 053411 (2019).

[55] P. Weiss, A. Cipris, M. O. Araújo, R. Kaiser, and W. Guerin, Phys. Rev. A 100, 033833 (2019).

[56] H. Breuer and F. Petruccione, Theory of Open Quantum Systems (Oxford University Press, Oxford, UK, 2002).

[57] C. Cohen-Tannoudji, J. Dupont-Roc, and G. Grynberg, Processus d'interaction entre photons et atomes (EDP Science/CNRS Éditions, Paris, 2001).

[58] R. Kosloff, J. Chem. Phys. 150, 204105 (2019).

[59] R. J. de Assis, T. M. de Mendonça, C. J. Villas-Boas, A. M. de Souza, R. S. Sarthour, I. S. Oliveira, and N. G. de Almeida, Phys. Rev. Lett. 122, 240602 (2019).

[60] N. Brunner, N. Linden, S. Popescu, and P. Skrzypczyk, Phys. Rev. E 85, 051117 (2012).

[61] C. L. Latune, I. Sinayskiy, and F. Petruccione, Sci. Rep. 9, 3191 (2019).

[62] M. A. Nielsen and I. L. Chuang, Quantum Computation and Quantum Information (Cambridge University Press, London, 2010).

[63] M. Esposito, K. Lindenberg and C. Van den Broeck, New J. Phys. 12, 013013 (2010).

[64] S. Deffner and E. Lutz, Phys. Rev. Lett. 107, 140404 (2011).

[65] C. L. Latune, I. Sinayskiy, and F. Petruccione, arXiv:1908.10384.

[66] A. Manatuly, W. Niedenzu, R. Román-Ancheyta, B. Çakmak, Ö. E. Müstecaplığlu, and G. Kurizki, Phys. Rev. E 99, 042145 (2019).

[67] D. Türkpençe and R. Román-Ancheyta, J. Opt. Soc. Am. B 36, 1252 (2019).

[68] J. P. Santos, L. C. Céleri, G. T. Landi, and M. Paternostro, npj Quantum Inf. 5, 23 (2019).

[69] R. Alicki, arXiv:1401.7865.

[70] R. Alicki and D. Gelbwaser-Klimovsky, New J. Phys. 17, 115012 (2015). 